\title{
Spin-Vacancy-Induced Long-Range Order in a New Haldane-Gap Antiferromagnet
}

\author{
Y. Uchiyama, ${ }^{1}$ Y. Sasago, ${ }^{1}$ I. Tsukada,${ }^{1}$ K. Uchinokura,,${ }^{1,2} *$ A. Zheludev,${ }^{3}$ T. Hayashi, ${ }^{4}$ N. Miura,${ }^{4}$ and P. Böni ${ }^{5}$ \\ ${ }^{1}$ Department of Applied Physics, The University of Tokyo, 6th Engineering Building, Bunkyo-ku, Tokyo 113-8656, Japan \\ ${ }^{2}$ Department of Advanced Materials Science, The University of Tokyo, 6th Engineering Building, \\ Bunkyo-ku, Tokyo 113-8656, Japan \\ ${ }^{3}$ Physics Department, Brookhaven National Laboratory, Upton, New York 11973-5000 \\ ${ }^{4}$ Institute for Solid State Physics, The University of Tokyo, 7-22-1 Roppongi, Minato-ku, Tokyo 106-8666, Japan \\ ${ }^{5}$ Laboratory for Neutron Scattering ETH \& PSI, CH-5232, Villigen PSI, Switzerland
}

(Received 12 April 1999)

\begin{abstract}
Magnetic susceptibility, high-field magnetization, and inelastic neutron scattering experiments are used to study the magnetic properties of a new $S=1$ quasi-one-dimensional antiferromagnet $\mathrm{PbNi}_{2} \mathrm{~V}_{2} \mathrm{O}_{8}$. Interchain interactions are shown to be almost, but not quite, strong enough to destroy the nonmagnetic singlet ground state and the energy gap in the magnetic excitation spectrum. Substituting nonmagnetic $\mathrm{Mg}^{2+}(S=0)$ ions for $\mathrm{Ni}^{2+}(S=1)$ induces a magnetically ordered state at low temperatures. To our knowledge this is the first observation of doping-induced long-range order in a Haldane-gap system.

PACS numbers: 75.30.Kz, 75.30.Hx, 75.40.Cx, 75.40.Gb
\end{abstract}

Unlike its classical counterpart, the quantum Heisenberg antiferromagnet (HAF) has a nonmagnetic spin-singlet ground state in one-dimensional (1D) space. A pioneering work by Haldane [1] led to a breakthrough in our understanding of this remarkable phenomenon, and of lowdimensional magnetism in general. Haldane suggested that the actual ground states are totally different for integerand half-integer-spin systems. The half-integer 1D HAF shows quasi-long-range order (power-law spin correlations). True long-range order can be induced by the slightest perturbations. In contrast, for integer spins the ground state singlet is robust. Intrachain spin correlations decay exponentially (true short-range order) and the excitation spectrum has a nonzero energy gap. The system remains nonmagnetic even in the presence of weak magnetic anisotropy or residual three-dimensional (3D) interactions. The spectacular properties of the integer-spin quantum HAF have been extensively studied theoretically and experimentally and are by now rather well understood. The current issue in quantum magnetism is how the singlet ground state is finally destroyed and long-range order (LRO) is formed when perturbations to the idealized Hamiltonian become sufficiently strong.

Among the factors that favor an ordered ground state are single-ion anisotropy and 3D interchain coupling in quasi-1D HAF systems. Increasing either of these parameters beyond some critical level leads to a quantum phase transition to a state with a Néel-like order parameter. This effect has been extensively studied and the corresponding phase diagram is known from numerical simulations [2]. As anticipated by Shender and Kivelson [3], another potential source of magnetic LRO is the introduction of spin vacancies in the quantum chains. Indeed, replacing some of the $S=1$ ions by $S=0$ sites severs the chains and liberates $S=1 / 2$ end-chain degrees of freedom [4]. Under certain circumstances these free spins can order in 3D space. To date, however, this phenomenon has not been observed in any material and is poorly understood theoretically. In the present paper we report the first observation of spin-vacancy-induced ordering in a new Haldane-gap compound $\mathrm{PbNi}_{2} \mathrm{~V}_{2} \mathrm{O}_{8}$, which sports a peculiar helical arrangement of magnetic ions and substantial (yet subcritical) interchain interactions.

The exact crystal structure of $\mathrm{PbNi}_{2} \mathrm{~V}_{2} \mathrm{O}_{8}$ has not been reported yet [5]. Preliminary x-ray diffraction measurements on powder samples prepared by conventional solidstate reaction suggest that this material is isomorphous with $\mathrm{SrNi}_{2} \mathrm{~V}_{2} \mathrm{O}_{8}$ [6] (Fig. 1 inset). $\mathrm{SrNi}_{2} \mathrm{~V}_{2} \mathrm{O}_{8}$ is tetragonal (space group $I 4_{1} c d$ ) with lattice parameters $a=$ 12.1617 $\AA$ and $c=8.3247 \AA$. We found that $\mathrm{PbNi}_{2} \mathrm{~V}_{2} \mathrm{O}_{8}$ has the same crystal symmetry and very similar lattice parameters: $a=12.249$ (3) $\AA$ and $c=8.354(2) \AA$. In the two isostructural compounds slightly distorted $\mathrm{NiO}_{6}$ octahedra are edge shared around the fourfold screw axis along the $c$ direction. All the $S=1 \mathrm{Ni}^{2+}$ ions, as well as all the nearest neighbor $\mathrm{Ni}^{2+}-\mathrm{Ni}^{2+}$ bonds are equivalent. The screw chains are separated by $\mathrm{VO}_{4}$ tetrahedra and $\mathrm{Sr}^{2+}$ or $\mathrm{Pb}^{2+}$ ions. Intrachain nearest-neighbor $\mathrm{Ni}-\mathrm{Ni}$ antiferromagnetic interactions are expected to dominate. The $\mathrm{V}^{5+}$ sites are presumed nonmagnetic.

To characterize the new $\mathrm{Pb}$-based system, bulk magnetic measurements were performed on highly aligned polycrystalline samples. These were prepared by applying a magnetic field of $9 \mathrm{~T}$ at room temperature to a mixture of $\mathrm{PbNi}_{2} \mathrm{~V}_{2} \mathrm{O}_{8}$ powder and epoxy resin (Stycast 1266). The Ni chains tend to align themselves along the applied magnetic field. A $c$-axis alignment of better than $3^{\circ}$ (FWHM) was achieved, as determined from measuring the rocking curve of the 004 Bragg reflection. Magnetic susceptibility was measured with a commercial SQUID magnetometer. The experimental $\chi(T)$ curves, shown in Fig. 1, have a broad maximum around $120 \mathrm{~K}$ 


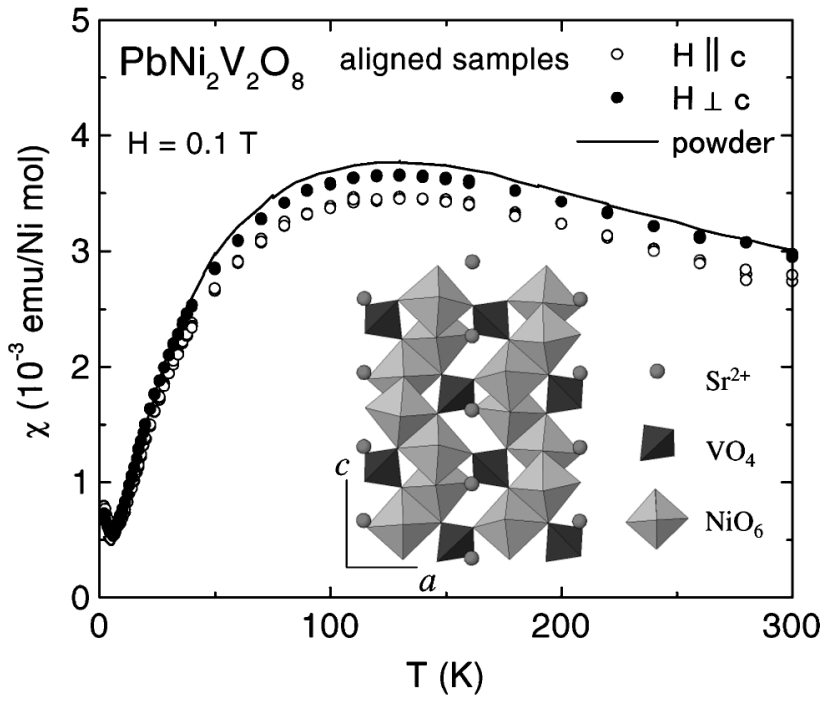

FIG. 1. Temperature dependence of magnetic susceptibility measured in $\mathrm{PbNi}_{2} \mathrm{~V}_{2} \mathrm{O}_{8}$. The solid line shows data for a nonaligned powder sample. The symbols are for highly aligned polycrystalline samples. The magnitudes of data for aligned samples are not exact because we cannot exactly estimate the quantity of the powder contained in the resin. True magnitude for $\boldsymbol{H} \| \boldsymbol{c}$ should be larger than that for $\boldsymbol{H} \perp \boldsymbol{c}$ because of the $c$-axis alignment. Inset: A schematic view of the crystal structure of $(\mathrm{Sr} / \mathrm{Pb}) \mathrm{Ni}_{2} \mathrm{~V}_{2} \mathrm{O}_{8}$.

for both $\boldsymbol{H} \perp \boldsymbol{c}$ and $\boldsymbol{H} \| \boldsymbol{c}$. Below this temperature, $\chi(T)$ decreases exponentially to almost zero. The residual low- $T$ susceptibility can be attributed to the presence of paramagnetic impurities. A straightforward analysis of the data collected in the range $0<T<40 \mathrm{~K}$ yields the thermal activation energies: $\Delta=29.4 \mathrm{~K}=2.53 \mathrm{meV}$ for $\boldsymbol{H} \| \boldsymbol{c}$, and $\Delta=27.8 \mathrm{~K}=2.39 \mathrm{meV}$ for a transverse field, respectively. In addition to the susceptibility measurements, high-field magnetization data were taken on an aligned polycrystalline sample in magnetic fields of up to $40 \mathrm{~T}$, using the pulsed magnetic facility at Institute for Solid State Physics. Figure 2 shows magnetization curves measured at $T=4.2 \mathrm{~K}$. At high fields an abrupt change of slope in $M(H)$ is observed for both $\boldsymbol{H} \| \boldsymbol{c}$ and $\boldsymbol{H} \perp \boldsymbol{c}$. The corresponding critical fields are $H_{c}^{(\perp)}=14 \mathrm{~T}$ and $H_{c}^{(\|)}=19 \mathrm{~T}$.

The activated temperature dependence of magnetic susceptibility is a clear signature of a nonmagnetic ground state. The distinct chain arrangement of magnetic $S=$ $1 \mathrm{Ni}^{2+}$ ions in the crystal structure suggests that we are in fact dealing with a Haldane antiferromagnet. The behavior of high-field magnetization is also typical of a Haldanegap system [7]. The starting point for describing the magnetism of $\mathrm{PbNi}_{2} \mathrm{~V}_{2} \mathrm{O}_{8}$ should therefore be a nearestneighbor Heisenberg Hamiltonian with a possible uniaxial single-ion anisotropy (tetragonal crystal structure): $H=J \sum_{i} \mathbf{S}_{i} \cdot \mathbf{S}_{i+1}+D \sum_{i}\left(S_{i}^{z}\right)^{2}$. For not too large $D$ the ground state is a Haldane singlet. The lowest energy excitations are a single longitudinal mode and a

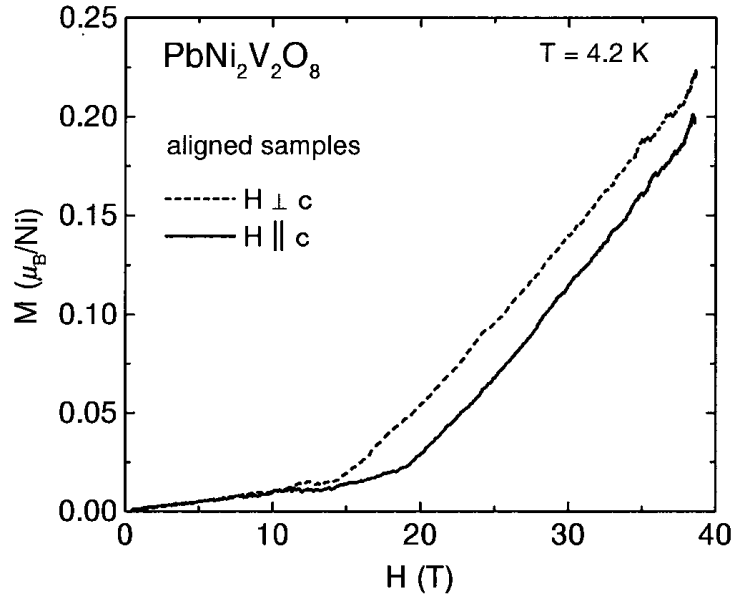

FIG. 2. Magnetization curves measured in $\mathrm{PbNi}_{2} \mathrm{~V}_{2} \mathrm{O}_{8}$ at $T=$ $4.2 \mathrm{~K}$ for magnetic fields parallel and perpendicular to the chain axis.

doublet of transverse-polarized Haldane excitations. Using Eq. (2.14) in Ref. [8], relating the critical fields $H_{c}^{(\perp)}$ and $H_{c}^{(\| l)}$ to the doublet and singlet gap energies $\Delta_{\perp}$ and $\Delta_{\|}$, for $\mathrm{PbNi}_{2} \mathrm{~V}_{2} \mathrm{O}_{8}$, we obtain $\Delta_{\perp}=2.2 \mathrm{meV}$ and $\Delta_{\|}=$ $1.2 \mathrm{meV}$. The intrachain exchange constant $J$ can be deduced from the high-temperature part of the measured $\chi(T)$ curves [9]. For $\mathrm{PbNi}_{2} \mathrm{~V}_{2} \mathrm{O}_{8}$ this analysis yields $J=$ $95 \mathrm{~K}=8.2 \mathrm{meV}$. Already we see that the mean gap energy $\left(2 \Delta_{\perp}+\Delta_{\|}\right) / 3=1.87 \mathrm{meV}$ is substantially smaller that the value $0.41 \mathrm{~J} \approx 3.36 \mathrm{meV}$, expected for noninteracting chains $[2,8]$. The most likely source of this discrepancy is substantial interchain exchange interactions. In their presence the excitation energies become dependent on the momentum transfer perpendicular to the chain axis. $\Delta_{\perp}$ and $\Delta_{\|}$in this case represent the global minima in the 3D dispersion and cannot be uniquely related to $J$.

Dynamic spin correlations in $\mathrm{PbNi}_{2} \mathrm{~V}_{2} \mathrm{O}_{8}$ were studied in a series of inelastic neutron scattering experiments on a $10 \mathrm{~g}$ powder sample at the DrüchaL 3-axis spectrometer (SINQ spallation source, PSI, Switzerland). Neutrons of fixed final energy $E_{f}=8 \mathrm{meV}$ were used with a (guide)-80'-80'-(open) collimation setup. An entire series of constant- $E$ scans was collected in the energy range 2-8 meV to map out the low-energy part of the magnetic dynamic structure factor $S(Q, \omega)$. Additional data were collected in constant- $Q$ scans. A typical data set measured at a momentum transfer just exceeding the 1D antiferromagnetic zone center $Q_{\pi}=4 \pi / c$ at $T=2 \mathrm{~K}$ is shown in the inset in Fig. 3. This and all other const- $Q$ scans clearly show the presence of a spin gap of roughly $3.5 \mathrm{meV}$. The main panel in Fig. 3 shows a constant- $E$ scan measured at $\hbar \omega=4 \mathrm{meV}$, i.e., just above the gap energy. In order to analyze these data we have calculated the powder average of the 3D dynamic magnetic structure factor for an isolated screw-shaped Haldane chain, as appropriate for $\mathrm{PbNi}_{2} \mathrm{~V}_{2} \mathrm{O}_{8}$ [10]. The relevant parameters 


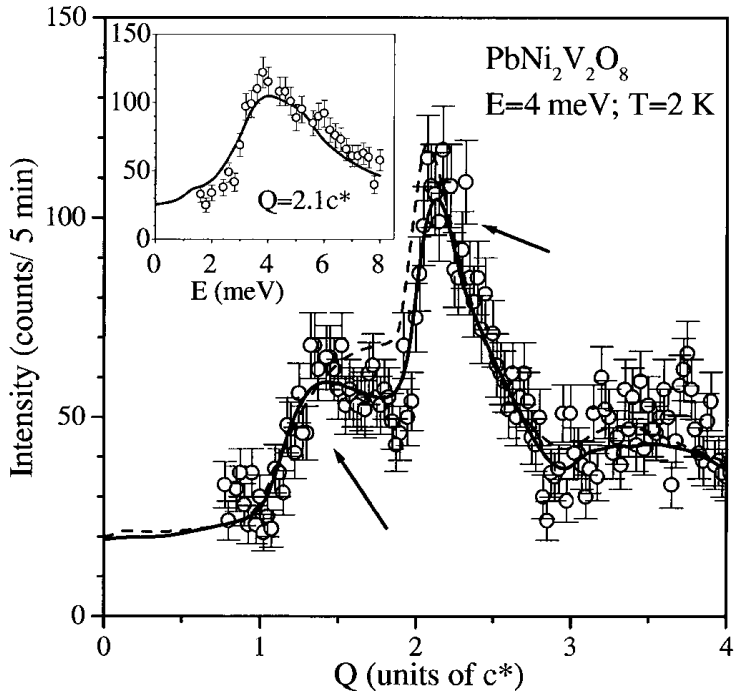

FIG. 3. Typical constant- $Q$ (inset) and constant- $E$ (main panel) inelastic neutron scattering scans measured in $\mathrm{PbNi}_{2} \mathrm{~V}_{2} \mathrm{O}_{8}$ powder at $T=2 \mathrm{~K}$. Lines are model fits, as described in the text.

of this cross section are the two gap energies $\Delta_{\perp}$ and $\Delta_{\|}$. Although this model (dashed line in Fig. 3) can reproduce the measured const- $Q$ scans fairly well and predicts the asymmetric feature at $Q_{\pi}$, it cannot account for the sharp peak seen at $Q \approx 3 \pi / c$ in constant $E$ scans.

A better agreement with the experiment could be obtained only by taking into account interchain interactions. We assumed a weak Heisenberg coupling between nearestneighbor Ni chains and treated it on the RPA level. A detailed description of this analysis is beyond the scope of this Letter and will be published elsewhere [10]. Compared to the noninteracting-chain model only one additional parameter, namely, the interchain coupling constant $J_{\perp}$ is introduced. The total number of variables is minimized by fixing the lowest excitation energies to the gap values deduced from high-field measurements. An excellent global fit to the data is obtained with $J_{\perp}=0.096 \pm$ $0.003 \mathrm{meV}$ from least squares refinement (solid lines in Fig. 3). From this value and the measured critical fields the intrinsic gap energies for noninteracting chains can be determined: $\Delta_{\perp}^{(0)}=4.1 \mathrm{meV}$ and $\Delta_{\|}^{(0)}=3.7 \mathrm{meV}$, respectively. Using the numerical results from Ref. [8], for the intrachain coupling constant $J$ and the anisotropy parameter $D$ we obtain $J=9.5 \mathrm{meV}$ and $D=-0.23 \mathrm{meV}$ (easy axis), respectively. The former value is in reasonably good agreement with the high- $T$ susceptibility estimate $J=8.2 \mathrm{meV}$.

The results presented above enable us to place the new material on the Sakai-Takahashi $D-J_{\perp}$ phase diagram [2]. For $\mathrm{PbNi}_{2} \mathrm{~V}_{2} \mathrm{O}_{8}$ we have $D / J \approx-0.024, J_{\perp} / J=$ 0.01 , and $z=4, z$ being the coordination number for interchain interactions. Using Fig. 8 in Ref. [2], we find that $\mathrm{PbNi}_{2} \mathrm{~V}_{2} \mathrm{O}_{8}$ is positioned in the Haldane phase, just below the borderline with the ordered Ising-like phase. In other words, by sheer chance the system is almost critical towards the formation of long-range order. As will be reported elsewhere [11], unlike $\mathrm{PbNi}_{2} \mathrm{~V}_{2} \mathrm{O}_{8}$, the isostructural $\mathrm{SrNi}_{2} \mathrm{~V}_{2} \mathrm{O}_{8}$ system falls on the opposite side of the transition line and is actually ordered in 3D space in a weak-ferromagnetic structure below $T_{N}=7 \mathrm{~K}$. A simple substitution of nonmagnetic $\mathrm{Sr}$ for nonmagnetic $\mathrm{Pb}$ slightly alters the coupling geometry, increasing interchain interactions or single-ion anisotropy beyond the critical values.

Having characterized the pure $\mathrm{PbNi}_{2} \mathrm{~V}_{2} \mathrm{O}_{8}$ compound, we now turn to the main subject of this paper, describing the emergence of Néel-like magnetic LRO upon the introduction of spin vacancies in this Haldane-gap system. Nonmagnetic sites are introduced into the spin chains by substituting $S=0 \mathrm{Mg}^{2+}$ ions for the $S=1 \mathrm{Ni}^{2+}$. The measured temperature dependences of magnetic susceptibility for a series of $\mathrm{Pb}\left(\mathrm{Ni}_{1-x} \mathrm{Mg}_{x}\right)_{2} \mathrm{~V}_{2} \mathrm{O}_{8}$ powder samples with $x$ ranging from 0 to 0.120 are shown in Fig. 4. Except for the $x=0$ compound, an increase of susceptibility is observed at low $T$ in all samples studied. The enhancement scales with $\mathrm{Mg}$ concentration and can be attributed to the presence of free $S=1 / 2$ spins at the ends of the Ni chains severed by the spin vacancies [12]. In this scenario the number of liberated spins is twice the number of substitutions. To check this scaling we compared the temperature dependence of magnetic susceptibility in $\mathrm{Mg}^{2+}(S=0)$ - and $\mathrm{Cu}^{2+}(S=1 / 2)$-substituted $\mathrm{PbNi}_{2} \mathrm{~V}_{2} \mathrm{O}_{8}$. The replacement of a $\mathrm{Ni}^{2+}$ ion by a $\mathrm{Cu}^{2+}$ ion also produces two end-chain spins. However, these two spins couple antiferromagnetically to the $\mathrm{Cu}$ spin, producing a ground state configuration of total $S=1 / 2$. The total number of added free spins in this case is equal to

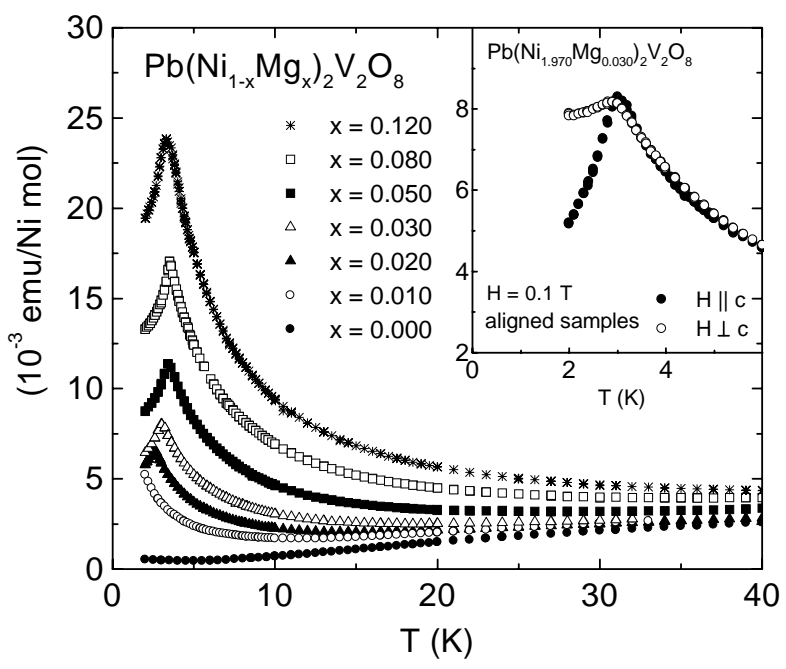

FIG. 4. Temperature dependences of magnetic susceptibility measured in $\mathrm{Pb}\left(\mathrm{Ni}_{1-x} \mathrm{Mg}_{x}\right)_{2} \mathrm{~V}_{2} \mathrm{O}_{8} \quad(0 \leq x \leq 0.120)$ powder samples. The inset shows the data for a highly aligned $\mathrm{Pb}\left(\mathrm{Ni}_{0.970} \mathrm{Mg}_{0.030}\right)_{2} \mathrm{~V}_{2} \mathrm{O}_{8}$. 
the total number of added $S=1 / 2$ ions. Experimentally, the measured $\chi(T)$ curve for the $2 \%$-Cu-doped $\mathrm{PbNi}_{2} \mathrm{~V}_{2} \mathrm{O}_{8}$ sample was found to be virtually indistinguishable from that for a $1 \%-\mathrm{Mg}$-doped sample.

The central result of this work is the observation of a sharp decrease in the magnetic susceptibility of $\mathrm{Pb}\left(\mathrm{Ni}_{1-x} \mathrm{Mg}_{x}\right)_{2} \mathrm{~V}_{2} \mathrm{O}_{8}$ below $T_{c} \approx 3.5 \mathrm{~K}$ for $x \geq 0.020$. The slope of $\chi(T)$ changes abruptly with reversing its sign. This behavior is a clear indication of a magnetic phase transition. Its nature is revealed in experiments on an aligned powder sample with $x=0.030$ (Fig. 4 inset). In this case the transition point is about $T_{c}=3.0 \mathrm{~K}$. Below this temperature a large anisotropy of susceptibility is observed. For $\boldsymbol{H} \| \boldsymbol{c}, \chi(T)$ decreases abruptly below $T_{c}$. In contrast, for $\boldsymbol{H} \perp \boldsymbol{c}$ it remains practically constant. This behavior is typical of a Néel antiferromagnet with spins ordering along the $c$ direction; that, as we have shown previously, is the magnetic easy axis of the system.

The effect of spin-vacancy substitution has been studied experimentally (see, for example, Ref. [12] and theoretically [4]) for a number of Haldane-gap systems. Apart from the appearance of free end-chain spins and the resulting paramagnetic contribution to the low- $T$ susceptibility, severing the chains leads to a reduction of the dynamic spin correlation length. $\mathrm{PbNi}_{2} \mathrm{~V}_{2} \mathrm{O}_{8}$ is, to our knowledge, the first example of a Haldane AF that orders magnetically upon doping. The key, of course, is in the interchain interactions: to order magnetically the liberated end-chain spins need to be coupled in 3D space. As we have demonstrated, interchain coupling is quite substantial in $\mathrm{PbNi}_{2} \mathrm{~V}_{2} \mathrm{O}_{8}$. The possibility of vacancy-induced LRO in a Haldane-gap system was first qualitatively discussed by Shender and Kivelson [3]. In particular, the authors suggested that there is a critical impurity concentration below which LRO does not occur. As to this point more detailed low- $T$ studies are required.

The magnetic transition that we observe in $\mathrm{Pb}\left(\mathrm{Ni}_{1-x} \mathrm{Mg}_{x}\right)_{2} \mathrm{~V}_{2} \mathrm{O}_{8}$ should not be confused with that in the extensively studied Haldane system $\mathrm{CsNiCl}_{3}$ $[13,14]$. Unlike in $\mathrm{PbNi}_{2} \mathrm{~V}_{2} \mathrm{O}_{8}$, direct interchain interactions in $\mathrm{CsNiCl}_{3}$ are stronger than the critical value, and even the undoped material has a magnetically ordered ground state. In fact, the introduction of $\mathrm{Mg}^{2+}$ vacancies leads to a decrease of the ordering temperature [15], in striking contrast with the behavior of $\mathrm{PbNi}_{2} \mathrm{~V}_{2} \mathrm{O}_{8}$. Rather, the situation in $\mathrm{Mg}$-doped $\mathrm{PbNi}_{2} \mathrm{~V}_{2} \mathrm{O}_{8}$ is similar to that in the spin-Peierls $\mathrm{CuGeO}_{3}$, doped with $\mathrm{Zn} \mathrm{[16],}$ $\mathrm{Mg}$ [17], or $\mathrm{Ni}$ [18], and the two-leg ladder system $\mathrm{SrCu}_{2} \mathrm{O}_{3}$ doped with $\mathrm{Zn}$ [19]. Although these materials are half-integer-spin antiferromagnets, their ground states fall in the same universality class as the Haldane state, and they both have a gapped magnetic excitation spectrum. Just as we propose for $\mathrm{PbNi}_{2} \mathrm{~V}_{2} \mathrm{O}_{8}$, the onset of long-range order is believed to be caused by interchain interactions that couple free spins liberated by impurity substitution. Doping-induced long-range magnetic order thus appears to be a universal feature of quasi-1D singlet quantum antiferromagnets with the excitation gap.

In summary, we have shown that $\mathrm{PbNi}_{2} \mathrm{~V}_{2} \mathrm{O}_{8}$ is a Haldane-gap system with substantial interchain interactions and is almost critical towards the formation of long-range order. For the first time long-range magnetic ordering was induced in a Haldane antiferromagnet by spin-vacancy substitution on the $S=1$ sites.

We thank T. Masuda for the cooperation in the various aspects of this work. This work was supported in part by Grant-in-Aid for COE Research "Phase Control of Spin-Charge-Photon Coupled System" from the Ministry of Education, Science, Sports, and Culture. Work at Brookhaven National Laboratory was carried out under Contract No. DE-AC02-98CH10886, Division of Material Science, U.S. Department of Energy.

*Author to whom correspondence should be addressed. Email address: uchinokura@ap.t.u-tokyo.ac.jp

[1] F. D. M. Haldane, Phys. Lett. 93A, 464 (1983); Phys. Rev. Lett. 50, 1153 (1983).

[2] T. Sakai and M. Takahashi, Phys. Rev. B 42, 4537 (1990).

[3] E. F. Shender and S. A. Kivelson, Phys. Rev. Lett. 66, 2384 (1991).

[4] I. Affleck, T. Kennedy, E. H. Lieb, and H. Tasaki, Phys. Rev. Lett. 59, 799 (1987); Commun. Math. Phys. 115, 477 (1988).

[5] B. Wanklyn, J. Mater. Sci. 13, 89 (1978).

[6] R. Wichmann and Hk. Müller-Buschbaum, Rev. Chim. Miner. 23, 1 (1986).

[7] K. Katsumata et al., Phys. Rev. Lett 63, 86 (1989); Z. Honda, H. Asakawa, and K. Katsumata, Phys. Rev. Lett. 81, 2566 (1998).

[8] O. Golinelli, Th. Jolicœur, and R. Lacaze, J. Phys. Condens. Matter 5, 7847 (1993).

[9] V. Gadet et al., Phys. Rev. B 44, 705 (1991).

[10] A. Zheludev et al. (to be published).

[11] Y. Uchiyama et al. (to be published).

[12] M. Hagiwara et al., Phys. Rev. Lett. 65, 3181 (1990).

[13] W. J. L. Buyers et al., Phys. Rev. Lett. 56, 371 (1986); R. M. Morra et al., Phys. Rev. B 38, 543 (1988).

[14] K. Kakurai et al., J. Phys. (Paris), Colloq. 49, C8-1433 (1988).

[15] S. S. Sosin et al., JETP Lett. 85, 114 (1997).

[16] M. Hase, I. Terasaki, and K. Uchinokura, Phys. Rev. Lett. 70, 3651 (1993); M. Hase et al., Phys. Rev. Lett. 71, 4059 (1993); Physica (Amsterdam) 215B, 146 (1995).

[17] T. Masuda et al., Phys. Rev. Lett. 80, 4566 (1998).

[18] N. Koide et al., cond-mat/9805059.

[19] M. Azuma et al., Phys. Rev. B 55, R8658 (1997). 